\title{
Adult liver transplant for hepatocellular carcinoma at Wits Donald Gordon Medical Centre in Johannesburg, South Africa
}

\author{
M Dempster, ${ }^{1}$ C Bouter, ${ }^{2}$ H Maher, ${ }^{2}$ P Gaylard, ${ }^{3}$ H Etheredge, ${ }^{2,4}$ J Fabian, ${ }^{2,4}$ A Mohamed, ${ }^{4,5} \mathrm{~J} \mathrm{Botha}^{5,6}$ \\ 1 Department of Internal Medicine, School of Clinical Medicine, Faculty of Health Sciences, Charlotte Maxeke Johannesburg \\ Academic Hospital, Johannesburg, South Africa \\ 2 Wits Donald Gordon Medical Centre, Johannesburg, South Africa \\ 3 Data Management and Statistical Analysis, University of the Witwatersrand, Johannesburg, South Africa \\ ${ }^{4}$ Department of Internal Medicine, School of Clinical Medicine, Faculty of Health Sciences, University of the Witwatersrand, \\ Johannesburg, South Africa \\ 5 Transplant Unit, Wits Donald Gordon Medical Centre, Johannesburg, South Africa \\ ${ }^{6}$ Department of Surgery, School of Clinical Medicine, Faculty of Health Sciences, University of the Witwatersrand, \\ Johannesburg, South Africa
}

Corresponding author: DrJ Fabian (june.fabian@mweb.co.za)

Background: For those with unresectable hepatocellular carcinoma, liver transplantation is considered the treatment of choice. Since 2006, the transplant programme at Wits Donald Gordon Medical Centre (WDGMC) has offered liver transplantation for selected patients with hepatocellular carcinoma. While the number of patients transplanted was small, we are unaware of any published data from Southern Africa describing outcomes in this group of liver transplant recipients. The aim of this study was to describe our experience as a case series.

Methods: The records of all patients with HCC who underwent deceased donor liver transplantation between April 2006 and March 2018 were reviewed retrospectively. Data were extracted from transplant clinic patient files, histopathology and pathology laboratory reports and an existing database of all liver transplant recipients at WDGMC. Patient survival was calculated from the time of transplant and survival estimates were determined by the Kaplan-Meier method.

Results: Thirty-one liver transplants were reviewed. The most common causes of underlying liver disease were infectious, mostly hepatitis B virus, and diseases of lifestyle including alcoholic/non-alcoholic steatohepatitis. Median age at transplant, 57 years (IQR 44-65 years), was younger than observed internationally, but consistent with reports from Africa. Male recipients predominated, in keeping with published trends. Overall, outcomes were worse than expected but for recipients who were within the University of California at San Francisco (UCSF) criteria for transplantation; survival was comparable to previously published data.

Conclusions: Despite limitations, this is the first documented series of patients undergoing liver transplantation for HCC in South Africa and demonstrates that good results can be achieved in appropriately selected patients.

S Afr J Surg 2019;57(3)

http://dx.doi.org/10.17159/2078-5151/2019/v57n3a3067

\section{Introduction}

In the Global Burden of Disease Study conducted from 1990 to 2015 , liver cancer was the sixth most common tumour worldwide and the fourth most frequent cause of cancerrelated deaths. The majority of liver cancers were caused by hepatitis $\mathrm{B}$ virus (HBV) and hepatitis $\mathrm{C}$ virus (HCV) infection and alcohol use. In the case of HBV, these cancers are considered preventable with widespread vaccination programmes. The most common histological subtypes observed in primary liver cancer are hepatocellular carcinoma (HCC) followed by cholangiocarcinoma. ${ }^{1}$

In South Africa, available data from the National Cancer
Registry in 2014 combines incident liver and bile duct cancers. Together, these comprised $0.67 \%$ and $0.44 \%$ of all cancers observed in men and women, respectively. Most cases of HCC were diagnosed between 45 to 79 years, with men from the Black African population group most affected. ${ }^{2}$ Introduction of the HBV vaccine as a public health intervention to reduce the incidence of HCC was implemented by the National Department of Health in South Africa in 1994.

Today liver transplantation is considered the treatment of choice for patients with unresectable HCC. Early experience with this treatment modality for $\mathrm{HCC}$ was hampered by recurrence of tumour in the transplanted liver and dismal outcomes. $^{3,4}$ It was not until the publication of the Milan 
criteria in 1996, where patients with a single tumour $<5 \mathrm{~cm}$ in size, or up to 3 tumours with none being $>3 \mathrm{~cm}$ in size, were considered appropriate for transplantation. Favourable outcomes were demonstrated in this group of liver transplant recipients and their survival was similar to others undergoing liver transplantation for non-malignant indications. ${ }^{5}$ Subsequently, the Milan criteria have been criticised as being too stringent and a modest expansion of the criteria with similar outcomes has been proposed by the University of California, San Francisco group, known as the UCSF criteria (single tumour $<6.5 \mathrm{~cm}$ or up to 3 tumours with none being $>4.5 \mathrm{~cm}$ and a total tumour diameter $<8 \mathrm{~cm}^{6}$

Because of organ shortages, those with HCC who fulfil Milan or UCSF criteria at the time of listing for liver transplantation may become ineligible and drop off the list due to disease progression. To address this, international practice assigns a Model for End Stage Liver Disease (MELD) exception score of 22 points when potential recipients are waitlisted and various locoregional therapeutic (LRT) modalities have evolved to limit tumour growth while awaiting transplant. ${ }^{7}$ These include (i) radiofrequency ablation (RFA); (ii) trans-arterial chemo-embolisation (TACE); (iii) surgical resection (hepatectomy); (iv) selective internal radiation therapy (SIRT); (v) therapy that might include any combination of i-iv, depending on the site and accessibility of the $\mathrm{HCC}$ lesion $/ \mathrm{s}^{8}$

The Wits Donald Gordon Medical Centre (WDGMC) Liver Transplant Programme was established in 2004 in Johannesburg, South Africa. In our programme, listing for liver transplantation is equitable, based on need. Thus, all eligible patients are on the same waiting list irrespective of payer status and deceased donor organs are allocated on a 'sickest first' basis according to the MELD score. ${ }^{9}$ The selection of HCC candidates for deceased donor liver transplantation is based on the UCSF criteria, a MELD exception score of 22 points is applied at the time of listing, and listed candidates are offered appropriate LRT while on the waitlist. Since the inception of our liver transplant programme, relatively few patients with primary HCC have undergone liver transplantation. To date, we are unaware of published data from Southern Africa regarding the clinical profile and outcomes of this group of liver transplant recipients. Therefore, the aim of this case series was to describe our experience of liver transplantation for HCC at WDGMC.

\section{Methods}

This case series comprises all patients with HCC who underwent deceased donor liver transplantation between April 2006 and March 2018 at WDGMC. The definitive diagnosis of HCC was confirmed on histological assessment of the explanted liver at the time of transplant. Tumour size was determined to be within or beyond UCSF criteria based on the histological assessment of size and number of lesions in the explanted liver. The following recipient data were collected: age at transplant; gender; aetiology of liver disease; pre-transplant serum alpha-feto protein (AFP) level (ng/ $\mathrm{ml}$ ); type of LRT (categorised as: RFA, TACE, hepatectomy, SIRT); post-transplant survival (minimum follow-up of 6 months); disease recurrence after transplant; cause of death. Data were extracted from transplant clinic patient records, histopathology and pathology laboratory reports and an existing REDCap database of all liver transplant recipients at WDGMC. ${ }^{10}$ Patient survival was calculated from the time of transplant and survival estimates were determined by the Kaplan-Meier method. All analyses were carried out using SAS version 9.4 for Windows.

\section{Results}

During the study period, 364 adult and 150 paediatric liver transplants were performed. Of these 31 patients with histologically confirmed $\mathrm{HCC}$ received a deceased donor liver transplant. The clinical characteristics of this case series are summarised in Table 1. There was only one paediatric recipient who was 8 years old at the time of transplant and overall, the median age was 57 years (interquartile range 44 65 years).

Chronic HBV and HCV were the most common cause of underlying liver disease $(39 \% ; 12 / 31)$, followed by nonalcoholic /alcoholic steatohepatitis $(32 \% ; 10 / 31)$. At the time of listing of potential recipients, two thirds had a normal AFP $(<10 \mathrm{ng} / \mathrm{ml})$ and only three patients had an AFP $>1000 \mathrm{ng} /$ $\mathrm{ml}$. Thirteen patients $(42 \%)$ underwent some form of LRT to the liver prior to transplant. TACE was the preferred modality, being used either alone or in combination with RFA in $9 / 13$ patients. Two patients underwent hepatectomy, one of whom was treated with RFA when recurrence occurred after hepatectomy but prior to referral for transplant. Both patients underwent salvage liver transplant for recurrent tumour. There was one patient who received SIRT with trans arterial Yttrium 90 radio-embolisation.

Overall, the 1 and 5 year recipient survival was $77 \%(95 \%$ CI $57-88 \%)$ and $61 \%$ (95\% CI 40-76\%) respectively. Twentyfive $(81 \%)$ patients were deemed to be within UCSF criteria and the respective survival of this sub-group of patients at 1 and 5 years was $88 \%$ (95\% CI 67-96\%) and 72\% (95\% CI 48-87\%) (Figure 1). Four recipients were transplanted despite being outside the UCSF criteria for the following reasons (i) HCC rupture and uncontrolled bleeding - while in hospital a marginal donor organ was going to be discarded and this was offered to the recipient as a lifesaving procedure; (ii) LRT was administered by a clinical team in Omaha, USA to reduce tumour size to within UCSF criteria. However, the explanted graft revealed tumour lesions outside of UCSF criteria; (iii) LRT was administered to reduce tumour size to within UCSF criteria but extrahepatic disease was identified on the explant; and (iv) HCC was diagnosed on a background of adenomatous disease and on explant multiple lesions were identified outside of UCSF criteria.

Eleven recipients died. The most common cause of death was sepsis with $7 / 11$ patients succumbing to either a bacterial, fungal (aspergillus species) or viral (cytomegalovirus) infection. Three recipients died from HCC recurrence and one died from an unexplained intracranial haemorrhage. 
Table 1: Characteristics of deceased donor liver transplant recipients with HCC at WDGMC from $2006-2018$ (n=31)

\begin{tabular}{|c|c|}
\hline Characteristic & n (\%) \\
\hline Age at transplant (years): median (IQR; range) & 57 years ( $44-65$ years; $8-74$ years) \\
\hline Gender & male $26(84)$; female $5(16)$ \\
\hline \multicolumn{2}{|l|}{ Causes of end stage liver disease } \\
\hline \multicolumn{2}{|l|}{ Infections } \\
\hline Hepatitis B virus & $8(26)$ \\
\hline Hepatitis $\mathrm{C}$ virus & $4(13)$ \\
\hline \multicolumn{2}{|l|}{ Steatohepatitis } \\
\hline Non-alcohol related & $5(16)$ \\
\hline Alcohol related & $2(6)$ \\
\hline Combination of alcohol/non-alcohol related & $3(10)$ \\
\hline \multicolumn{2}{|l|}{ Other } \\
\hline Cryptogenic & $2(6)$ \\
\hline Haemochromatosis & $2(6)$ \\
\hline Progressive Familial Intrahepatic Cholestasis type 2 & $1(3)$ \\
\hline Primary biliary cirrhosis & $1(3)$ \\
\hline Auto-immune hepatitis & $1(3)$ \\
\hline Veno-occlusive disease (Budd-Chiari Syndrome) & $1(3)$ \\
\hline Multiple adenomatosis ${ }^{1}$ & $1(3)$ \\
\hline $\mathrm{UCSF}^{2}$ criteria & within criteria $25(81) /$ outside of criteria $4(13)$ \\
\hline unknown & $2(6)$ \\
\hline \multicolumn{2}{|l|}{ Serum $\mathrm{AFP}^{3}$ level pre-transplant $(\mathrm{ng} / \mathrm{ml})$} \\
\hline$<10$ & $19(61)$ \\
\hline$\geq 10<100$ & $6(19)$ \\
\hline$\geq 10<500$ & $2(6)$ \\
\hline$\geq 500<1000$ & $1(3)$ \\
\hline$\geq 1000$ & $3(10)$ \\
\hline No $\mathrm{LRT}^{4}$ received & $14(45)$ \\
\hline LRT received & $13(42)$ \\
\hline unknown & $4(13)$ \\
\hline
\end{tabular}

${ }^{1}$ Possibly arising from exogenous androgen administration, ${ }^{2}$ UCSF: University of California at San Francisco, ${ }^{3}$ AFP: Alpha-feto protein, ${ }^{4}$ LRT: Locoregional therapy

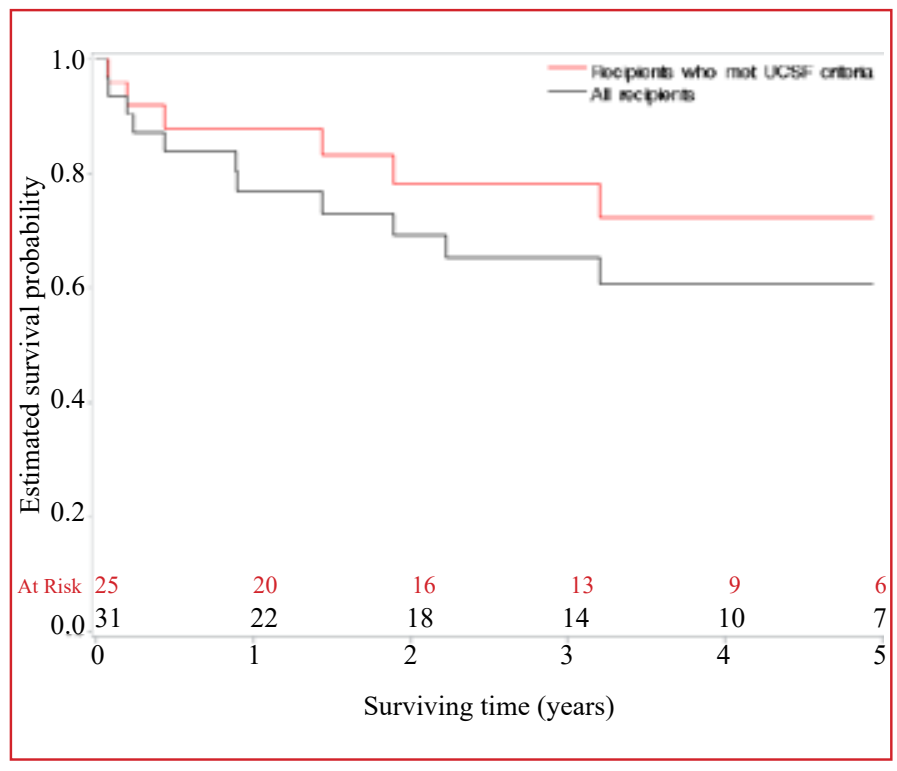

Figure 1: (Left) Survival of adult liver transplant recipients with HCC (overall, and by UCSF criteria)

Overall survival (percentage and $95 \%$ confidence limits):

90 days: $87 \%(69-95 \%) ; 1$ year: $77 \%(57-88 \%) ; 3$ years: $65 \%(45-80 \%) ; 5$ years: $61 \%(40-76 \%)$

Survival within UCSF criteria (percentage and 95\% confidence limits): 90 days: $92 \%$ (72-98\%); 1 year: $88 \%$ $(67-96 \%) ; 3$ years: $78 \%(55-90 \%) ; 5$ years: $72 \%$ $(48-87 \%)$

\section{Discussion}

In this case series, most liver transplant recipients were male which is concordant with the global predominance of men presenting with HCC. However, when compared with the global average age at diagnosis of 62 years, our recipients were younger with $61 \%$ aged $\leq 60$ years of age at time of transplantation. Therefore, they would have been diagnosed with $\mathrm{HCC}$ at an even earlier age, which is consistent with data 
reported from Africa. ${ }^{11}$ In keeping with published literature, we found the most common cause of underlying liver disease was chronic viral hepatitis, with hepatitis B predominating, followed by diseases of lifestyle such as non-alcoholic and alcoholic steatohepatitis. ${ }^{12,13}$ In those with HBV, lamivudine is administered in the immediate postoperative period and switched to tenofovir when renal function has normalised, usually within the first three months.

Contemporary studies confirm that serum AFP is a simple and reliable tumour marker that reflects the biological behaviour of HCC and thus the risk of metastasis. Recent evidence suggests that AFP levels might add prognostic information beyond the size and number of tumours. ${ }^{14,15}$ Differences in survival based on serum AFP levels have been clearly demonstrated. The UCSF group showed that recipients within Milan criteria who have a serum AFP greater than $1000 \mathrm{ng} / \mathrm{ml}$ at the time of transplant have a 50\% 5-year disease free survival compared to an $80 \%$ 5-year disease free survival for those with an AFP $<1000 \mathrm{ng} / \mathrm{ml} .{ }^{16}$ While the number of patients transplanted in this case series is too small for similar analyses, it is noteworthy that most of our recipients had normal or low AFP levels, which exceeds what has been observed elsewhere, where low AFP levels have occurred in up to $30 \%$ of cases. However, this could also represent a selection bias based on referral patterns to our transplant programme or possibly be due to tumours with lower potential risk of recurrence..$^{15}$

LRT has long been used in HCC patients for a variety of reasons; either to prevent tumour growth and keep listed patients within criteria, or to down-stage tumours that are beyond criteria, and lastly as neoadjuvant therapy to improve the outcome after transplant. ${ }^{17}$ While LRT has never been demonstrated to confer a survival benefit after transplant, emerging data suggests that response to LRT might predict outcome after liver transplant. A large, multi-centre European study, the aim of which was to study tumour response after LRT and change in AFP over time (as a prognostic indicator) after liver transplant, showed that when serum AFP increased by more than $15 \mathrm{ng} / \mathrm{ml} / \mathrm{month}$ with radiologic progression, 5-year survival worsened regardless of pretransplant status according to Milan criteria. ${ }^{18}$ Based on these data, a prudent clinical recommendation is that patients with serum AFP $>1000 \mathrm{ng} / \mathrm{ml}$ must demonstrate a decrease in serum AFP to $<500 \mathrm{ng} / \mathrm{ml}$ with LRT prior to being offered liver transplant, regardless of their Milan/UCSF status. In addition, we should create a more structured approach to LRT considering its potential to refine the selection of patients for liver transplantation.

In a large single-centre study from the USA, Duffy et al. demonstrated a marked improvement in survival for patients within Milan criteria. ${ }^{19}$ The United Network for Organ Sharing (UNOS) adopted these criteria into the allocation system for deceased donor organs in the USA. Initially HCC patients were assigned a disproportionately large number of points, ultimately disadvantaging waitlisted patients without HCC. The allocation system has undergone numerous refinements and patients with early stage $\mathrm{HCC}$ are now assigned 22 points under the MELD system. ${ }^{7}$ Our overall 1- and 5-year survival rates for the whole group are somewhat disappointing when compared to international studies, however when patients with tumours beyond UCSF criteria are excluded from analysis, the survival at 1 and 5 years is in keeping with that found in the literature. ${ }^{20-22}$

There are limitations to this case series namely, it is retrospective, from a single centre and the number of recipients is small, limiting the study to a descriptive analysis.

\section{Conclusion}

This case series describes the clinical profile and outcomes of patients with HCC undergoing deceased donor liver transplantation in our transplant programme at WDGMC. The predominance of men with $\mathrm{HCC}$ and the causes of underlying liver disease appears to coincide with global data, however our patients present at a younger age. No conclusions can be drawn from the low serum AFP levels that we observed but from evidence in emerging literature, we should implement serial serum AFP measurements in our clinical practice to refine our prognostic assessment for potential recipients on the waitlist. We used TACE as the most common form of LRT which is consistent with international standards; however it is also clear that we need to apply LRT not only for downstaging tumours that are beyond criteria but as a measure of the biological nature and metastatic potential of the tumour in order to refine our selection processes.

Despite the limitations of this retrospective study, this is the first published series of patients undergoing liver transplantation for $\mathrm{HCC}$ in South Africa. The outcomes demonstrate that good results can be achieved in appropriately selected patients. It is our hope that this form of therapy will be more widely offered to patients with HCC who may be potential liver transplant candidates.

\section{Ethics approval}

Human Research Ethics approval for this study was obtained from the University of Witwatersrand (M160367).

\section{Conflict of interest}

The authors have none to declare.

\section{Acknowledgment of funding}

Wits Donald Gordon Medical Centre provided the funding for the biostatistical analysis.

\section{REFERENCES}

1. Akinyemiju $\mathrm{T}$, Ahmed $\mathrm{M}$, Alam $\mathrm{N}$, et al. The Burden of Primary Liver Cancer and Underlying Etiologies from 1990 to 2015 at the Global, Regional, and National Level: Results From the Global Burden of Disease Study 2015. JAMA Oncol. 2017;3(12):1683-91. doi:10.1001/jamaoncol.2017.3055

2. National Cancer Registry. Cancer in South Africa. 2014. Available from: http://www.nicd.ac.za/centres/national-cancerregistry/http://www.nicd.ac.za/centres/national-cancer-registry/. Accessed Aug 22018.

3. Iwatsuki S, Gordon RD, Shaw B w J, Starzl TE. Role of Liver Transplantation in Cancer Therapy. Ann Surg. 1985;202(4):401. 
PMID: 2996449

4. Olthoff KM, Millis JM, Rosove M, Goldstein LI, Ramming K, Busuttil RW. Is liver transplantation justified for the treatment of hepatic malignancies? Arch Surg. 1990;125(10):1261-6; discussion 1266-8. doi:10.1001/archsurg.1990.01410220045007

5. Mazzaferro V, Regalia E, Doci R, et al. Liver transplantation for the treatment of small hepatocellular carcinomas in patients with cirrhosis. N Engl J Med. 1996;334(11):693-9. doi:10.1056/ NEJM199603143341104

6. Yao FY, Ferrell L, Bass NM, et al. Liver transplantation for hepatocellular carcinoma: expansion of the tumor size limits does not adversely impact survival. Hepatology. 2001;33(6):1394-403. doi:10.1053/jhep.2001.24563

7. Goldberg DS, Olthoff KM. Standardizing MELD Exceptions: Current Challenges and Future Directions. Curr Transplant Rep. 2014;1(4):232-7. doi:10.1007/s40472-014-0027-4

8. Schwartz M, Roayaie S, Uva P. Treatment of HCC in Patients Awaiting Liver Transplantation. Am J Transplant. 2007;7(8):1875-81. doi:10.1111/j.1600-6143.2007.01863.x

9. Singal AK, Kamath PS. Model for End-stage Liver Disease. J Clin Exp Hepatol. 2013;3(1):50-60. doi:10.1016/j. jceh.2012.11.002

10. Harris PA, Taylor R, Thielke R, Payne J, Gonzalez N, Conde JG. Research Electronic Data Capture (REDCap) - A metadatadriven methodology and workflow process for providing translational research informatics support. J Biomed Inform. 2009;42(2):377-81. doi:10.1016/j.jbi.2008.08.010

11. Yang JD, Altekruse SF, Nguyen MH, Gores GJ, Roberts LR. Impact of country of birth on age at the time of diagnosis of hepatocellular carcinoma in the United States. Cancer. 2017;123(1):81-9. doi:10.1002/cncr.30246

12. Perz JF, Armstrong GL, Farrington LA, Hutin YJF, Bell BP. The contributions of hepatitis $B$ virus and hepatitis $C$ virus infections to cirrhosis and primary liver cancer worldwide. J Hepatol. 2006;45(4):529-38. doi:10.1016/j.jhep.2006.05.013

13. Vento S, Dzudzor B, Cainelli F, Tachi K. Liver cirrhosis in subSaharan Africa: neglected, yet important. Lancet Glob Health. 2018;6(10):e1060-e1061. doi:10.1016/S2214-109X(18)30344-9
14. Clavien P-A, Lesurtel M, Bossuyt PMM, Gores GJ, Langer B, Perrier A. Recommendations for liver transplantation for hepatocellular carcinoma: an international consensus conference report. Lancet Oncol. 2012;13(1):e11-e22. doi:10.1016/S14702045(11)70175-9

15. Duvoux C, Roudot-Thoraval F, Decaens T, et al. Liver transplantation for hepatocellular carcinoma: a model including $\alpha$-fetoprotein improves the performance of Milan criteria. Gastroenterology. 2012;143(4):986-94. doi:10.1053/j. gastro.2012.05.052

16. Yao FY, Mehta N, Flemming J, et al. Downstaging of Hepatocellular Cancer before Liver Transplant: Longterm Outcome compared to Tumors within Milan Criteria. Hepatology. 2015;61(6):1968-77. doi:10.1002/hep.27752

17. Poon RT-P, Fan S-T, Tsang FH-F, Wong J. Locoregional Therapies for Hepatocellular Carcinoma: A Critical Review from the Surgeon's Perspective. Ann Surg. 2002;235(4):466-86. PMID: 11923602

18. Lai Q, Iesari S, Melandro F, Mennini G, Rossi M, Lerut $\mathrm{J}$. The growing impact of alpha-fetoprotein in the field of liver transplantation for hepatocellular cancer: time for a revolution. Transl Gastroenterol Hepatol. 2017;2. doi:10.21037/ $\operatorname{tgh} .2017 .09 .05$

19. Duffy JP, Vardanian AJ, Benjamin EC, et al. Liver transplantation criteria for hepatocellular carcinoma should be expanded: a 22-year experience with 467 patients at UCLA. Ann Surg. 2007;246(3):502-9; discussion 509-11. doi:10.1097/ SLA.0b013e318148c704

20. Doyle MBM, Vachharajani N, Maynard E, et al. Liver transplantation for hepatocellular carcinoma: long-term results suggest excellent outcomes. J Am Coll Surg. 2012;215(1):1928; discussion 28-30. doi:10.1016/j.jamcollsurg.2012.02.022

21. Hemming AW, Cattral MS, Reed AI, Van der Werf WJ, Greig PD, Howard RJ. Liver Transplantation for Hepatocellular Carcinoma. Ann Surg. 2001;233(5):652-9. PMID: 11323504

22. $\mathrm{Xu} \mathrm{X}, \mathrm{Lu} \mathrm{D}$, Ling $\mathrm{Q}$, et al. Liver transplantation for hepatocellular carcinoma beyond the Milan criteria. Gut. 2016;65(6):1035-41. doi:10.1136/gutjnl-2014-308513 